\title{
Limits for the determination of guiding values from intra-laboratory data basis, demonstrated by prostate specific antigen (PSA) ${ }^{1)}$
}

\section{Eberhard Gurrî,*, Friedrich Krönig², Anja Golbeck $^{3}$ and Farhad Arzideh ${ }^{4}$}

${ }^{1}$ Klinikum Links der Weser gGmbH, Bremen, Department for Clinical Chemistry and Central Laboratory, Germany

${ }^{2}$ Klinikum Oldenburg gGmbH, Clinic for Cardiac Surgery, Germany

${ }^{3}$ Klinikum Bremen Ost gGmbH, Central Laboratory, Germany

${ }^{4}$ University of Bremen, Institute of Statistics, Germany

\begin{abstract}
The German guidelines for early detection of prostate cancer propose the use of digital rectal palpation and prostate specific antigen (PSA). PSA levels above $4 \mu \mathrm{g} / \mathrm{L}$ are considered to be suspect. However, method comparison of six different assays resulted in differences up to $20 \%$ in the range of $4 \mu \mathrm{g} / \mathrm{L}$, although all assays used the Stanford 90/10 (WHO 96/670) calibration. Interpretation of PSA levels therefore has to take into consideration assay-specific characteristics, using assay-specific local reference ranges. However, the estimation of reference ranges by, e.g., analysis of frequency distribution failed with regard to the high prevalence of benign prostate hyperplasia. PSA velocity is proposed for early detection. Interpretation of PSA velocity especially at low PSA levels has to take into consideration analytical imprecision and biological variation.
\end{abstract}

Keywords: action limits; critical difference; frequency distribution; method comparison; standardisation.

1)Original German online version at: http://www.referenceglobal.com/doi/pdf/10.1515/JLM.2009.022.

The German article was translated by Compuscript Ltd. and authorized by the authors.

*Correspondence: Prof. Dr. Eberhard Gurr, Klinikum Links der Weser gGmbH, Department for Clinical Chemistry and Central Laboratory, Senator-Wessling-Str. 1, 28277 Bremen, Germany

Tel.: + 49 (0421) 8791670

Fax: + 49 (0421) 8791672

E-Mail: eberhard.gurr@klinikum-bremen-Idw.de

\section{Introduction}

PSA belongs to the group of tumour markers that are deemed to be of diagnostic significance in detecting tumours. The guidelines of the German Association of Urologists recommend an action limit of $4.0 \mu \mathrm{g} / \mathrm{L}$ for PSA in combination with a digital rectal examination. For PSA results above $4.0 \mu \mathrm{g} / \mathrm{L}$ and in the case of conspicuous palpitation findings, a biopsy is recommended to detect the presence of a tumour [1]. Various immunological procedures are used to determine the PSA concentration in peripheral blood. What all procedures have in common is that they are designed as sandwich assays. But the pairs of antibodies used differ from manufacturer to manufacturer [2]. In peripheral blood, there are up to seven different PSA populations in circulation, which are either enzymatically inactive or whose enzymatically active centre has been inhibited [3-5]. It is improbable that all antibody pairs would attach themselves equally to the antigenic epitopes of heterogeneous PSA populations. The PSA result must, therefore, be expected to be dependent on the method used. Following up on that, inter-lab tests have found, dependent on the manufacturer of each test, that they may differ by a factor of two [6]. It is doubtful that, under such conditions, it is possible to work with a uniform, method-independent reference range or action limit. Perhaps, each user may have to determine his own reference range or action limit for his procedure. A comparison of methods has been carried out for PSA analysis, to determine the reference range the method of frequency breakdown has been performed and critical difference to assess PSA progression rates have been calculated.

\section{Materials and methods}

For the purposes of comparing methods, PSA has been analysed in serum samples taken from 210 patients and test persons with seven different reagents by six different manufacturers (Table 1). All serum samples were stored at a temperature of $-35^{\circ} \mathrm{C}$ prior to the examination. Samples were stored no longer than eight weeks. The regression lines were determined according to Passing and Bablok [7].

The frequency distribution was broken down by means of a recently published statistical procedure for frequency breakdown $[8,9]$. The data on which the study was based had been 
Table 1 Comparison of different methods to determine PSA $(n=210)$. All manufacturers trace their calibration back to WHO 96/670 = Stanford 90/10.

\begin{tabular}{llll}
\hline & "Best fit" line & Action limit & \\
\cline { 3 - 4 } & & Estimated $^{2}$ & Stated $^{3}$ \\
\hline A Beckman Dxi & $1.0 x+0$ & 4.0 & 4.0 \\
B Beckman Dxi WHO & $0.806 x^{*}+0.038^{\star}$ & 3.26 & 3.1 \\
C Abbott Architect & $0.935 x^{*}-0.015$ & 3.73 & 3.9 \\
D Bayer Centaur & $0.808 x^{*}+0.009$ & 3.24 & 4.0 \\
E Brahms Kryptor & $0.959 x^{*}+0.095^{\star}$ & 3.93 & 4.0 \\
F DPC Immulite 2500 & $0.834 x^{*}+0.046^{*}$ & 3.38 & 3.2 \\
G Roche Elecsys 2010 & $0.962 x^{*}+0.138^{*}$ & 3.98 & 4.0 \\
\hline
\end{tabular}

${ }^{*}$ Significantly different; ${ }^{1}$ Regression lines according to Passing/Bablok; reference system A; ${ }^{2}$ Estimation of action limit using the regression equation derived from the method comparison ( $x=4 \mu \mathrm{g} / \mathrm{L})$; ${ }^{3}$ Manufacturers' information.

derived from 6833 PSA tests that had been carried out for patients at the Klinikum Bremen Mitte throughout one calendar year. Results of patients in intensive care were disregarded.

The critical difference for PSA was determined in consideration of the intra-individual biological variation $\mathrm{CV}_{1}$ and the analytical imprecision $\mathrm{CV}_{\mathrm{A}}$ in accordance with the following equation:

$\mathrm{CD}=2^{*} 2^{1 / 2 *}\left(\mathrm{CV}_{1}^{2}+\mathrm{CV}_{\mathrm{A}}^{2}\right)^{1 / 2}$

\section{Results}

\section{Comparison of methods}

All manufacturers traced their calibrations back to the standard WHO 96/670 (Stanford 90/10). Additionally, most manufacturers made an additional adaptation to the procedure that used to serve as a reference procedure in the past and on the basis of which many studies had been conducted (Hybritec PSA, procedures A, C, E, G). Procedure $A$ in this comparison of methods corresponded to Hybritec PSA and was therefore chosen as the reference method. Procedure $A$ has an action limit of $4.0 \mu \mathrm{g} / \mathrm{L}$. As for $\mathrm{C}, \mathrm{E}$ and $\mathrm{G}$, using the regression lines of the method comparison with $A$, it is possible to derive largely corresponding action limits (Table 1). Procedure B corresponds to $A$, but its calibration was not adapted to Hybritec PSA. For B, D and F, the regression lines show significantly lower action limits. Obviously calibration in this case had not been adapted for Hybritec PSA. As for $B$ and $F$, the manufacturers had stated lower action limits; with $\mathrm{D}$, however, there is a clear difference in the action limit between manufacturers' information and the result produced by this comparison.

Overall, it has been shown, as expected, that there is no uniform, method-independent action limit of $4 \mu \mathrm{g} / \mathrm{L}$.

\section{Breakdown of frequency distribution}

The frequency distribution curves indicate the presence of several populations. Even after applying an age stratification, the picture did not change much (Figure 1). The separation of a Gauss-distributed (normal) population

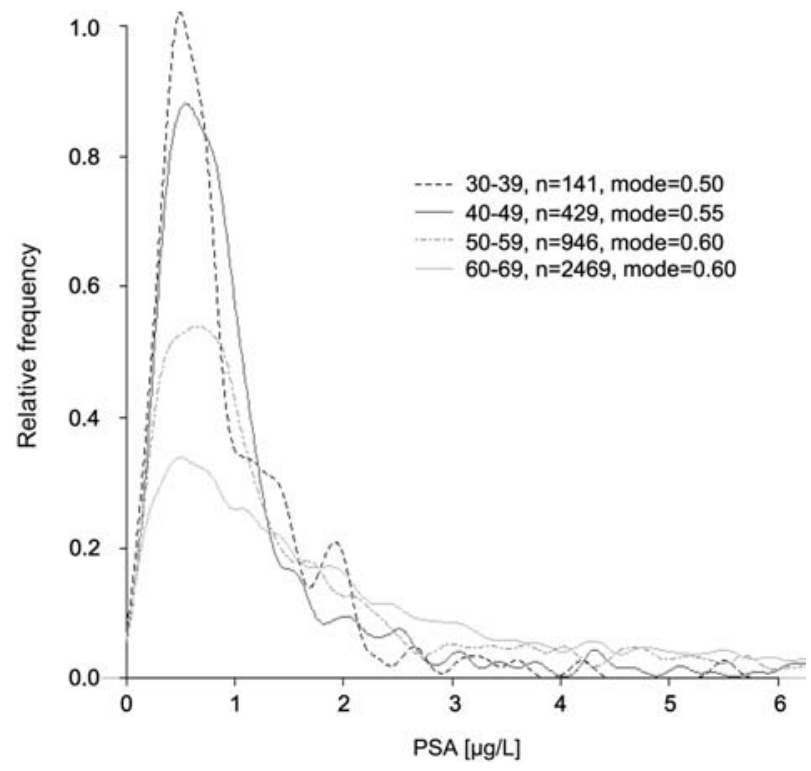

Figure 1 Frequency distribution of PSA values in a clinical study of men aged 30-69.

Mode: most frequent PSA value from each age group.

was not possible, both in the overall distribution and after age stratification and a Box-Cox transformation. The determination of the reference range or of an action limit by breaking down frequency distributions, as described for enzyme activities [10], failed when applied to PSA. The following arguments can be discussed as possible causes:

1. A breakdown into a normal and residual fraction requires that an area can be identified in the frequency distribution that can be used to extrapolate a Gauss distribution with sufficient certainty. The greater the prevalence of the pathological population, and the more the two populations overlap, the less accurate any separation of the normal distribution will be. Based on the overall distribution of the PSA levels studied here, it was also impossible - even after a transformation - to identify a Gauss distribution due to the considerable overlaps 
between the pathological and non-pathological populations.

2. The predictive value of an action limit is influenced by the proportion of wrongly classified patients. The more the healthy and the pathological populations overlap, the more difficult it is to find an action limit with satisfactory predictive values. Even if a frequency distribution can be broken down into a normal distribution and a pathological population, the action limit will still not provide a satisfactory distinction between healthy and pathological when populations overlap considerably. Such a diagnostic behaviour would also have to be expected for PSA. After all, $20 \%$ of patients with prostate cancer have PSA levels below $4 \mu \mathrm{g} / \mathrm{L}$, while only $50 \%$ of patients with PSA levels of $10 \mu \mathrm{g} / \mathrm{L}$ suffer from prostate cancer [11-14].

3. The action limit also remains diagnostically unsatisfactory when the pathological population to be determined represents only a small subgroup of all pathological values. As a result of the prevalence of prostate cancer and benign prostatic hyperplasia, increased levels are most frequently due to benign prostatic hyperplasia $[15,16]$.

In the case of PSA, all of the above causes prevent the definition of a valid decision limit: the frequency distribution cannot be broken down; the prevalence of benign hyperplasia exceeds that of the prostate cancer by a multiple factor, and the overlap in the PSA levels of the healthy and pathological populations is so great that any diagnostic statement would not be satisfactory.

\section{PSA velocity over time}

The absence of a valid action limit for the detection of prostate cancer and the method-dependency of PSA tests have resulted in the diagnostic use of intra-individual PSA increases over time [1]. For such purpose, increases of $0.75 \mu \mathrm{g} / \mathrm{L}$ per year are deemed to indicate the presence of a carcinoma. How high an increase must be so that the new result is analytically and significantly different from the previous value is governed by the critical difference (CD). A CV 1 of $18 \%$ is stated for PSA [17]. Own studies involving four test persons and seven different methods have consistently produced variation coefficients below 11\% [18]. By nature, the critical difference depends on the PSA concentration (Figure 2). Assuming a $\mathrm{CV}_{1}$ of $10 \%$, an increase of $0.75 \mu \mathrm{g} / \mathrm{L}$, with an analytical imprecision of $2 \%$, can be identified with certainty only for PSA levels under $3 \mu \mathrm{g} / \mathrm{L}$. With an analytical imprecision of $10 \%$, the same increase can be detected against analytical or biological fluctuations only for initial values below $2.2 \mu \mathrm{g} / \mathrm{L}$. In the case of an initial value of $4 \mu \mathrm{g} / \mathrm{L}$, the current value must be at least $5.0 \mu \mathrm{g} / \mathrm{L}$ with an analytical imprecision of $2 \%$ and $5.5 \mu \mathrm{g} / \mathrm{L}$ with an analytical imprecision of $10 \%$ in order to detect a significant increase.

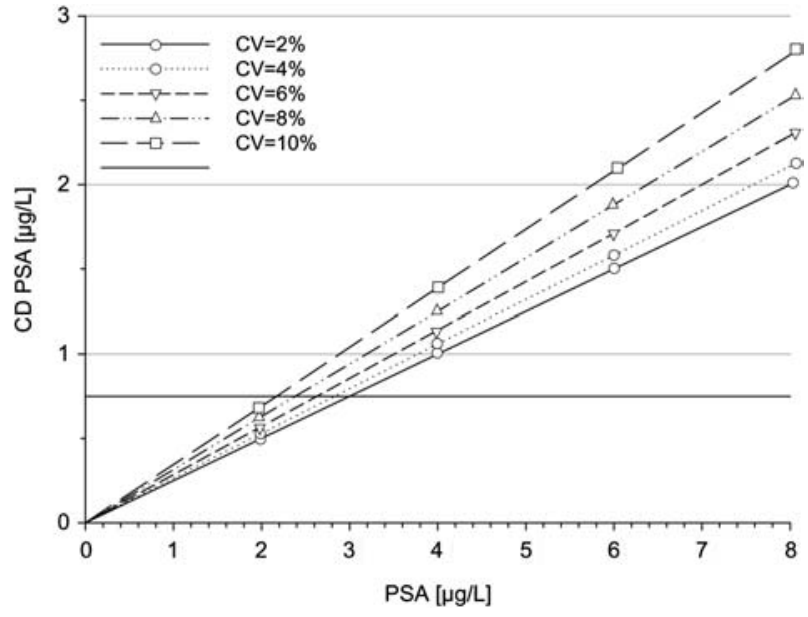

Figure 2 Critical difference (CD) in relation to PSA concentration.

An intra-individual biological variation of $10 \%$ has been assumed.

\section{Discussion}

Although PSA is one of the few tumour markers that are recommended for tumour detection, its diagnostic value is still controversial - due to poor tumour specificity: Benign prostatic hyperplasia, like prostate cancer, occurs with PSA increases and has a much higher prevalence [15]. But if PSA cannot be used to differentiate between benign hyperplasia and cancer, all methods must ultimately fail that are used to attempt to define action limits for PSA with sufficiently high predictive values. Of course, this is also true of the method of breaking down populations, as applied here.

In analytical terms, the various methods to measure PSA are equally satisfactory, in spite of different antibody pairs, because all manufacturers use calibrators that are based on the same reference material. The discrepancies identified in inter-lab tests have not been found in the patient samples examined here. Since the control samples have been enhanced with seminal plasma, the difference between patient and control samples is most likely due to the influence of PSA forms not occurring in peripheral blood [19].

Complications in determining action limits occur as a result of the additional adaptation for an "old" reference method. Manufacturers do not always state their traceability completely, and the action limit is not always set correctly in relation to the standardisation.

It may, therefore, be helpful for diagnostic purposes to evaluate PSA levels over time. The detection of significant increases requires knowledge of analytical imprecision and intra-individual biological variation in order to assess the critical difference. If one assumes a biological variation of $10 \%$, an increase of $0.75 \mu \mathrm{g} / \mathrm{L}$, which gives rise to suspicion of tumour, can be detected only for initial values up to $3.0 \mu \mathrm{g} / \mathrm{L}$ if the analytical imprecision is $<2 \%-$ a level of imprecision that is difficult to achieve 
even today. With an imprecision of $6 \%$ and an initial value of $4.0 \mu \mathrm{g} / \mathrm{L}$, the PSA concentration must have risen to over $5.2 \mu \mathrm{g} / \mathrm{L}$ to produce a significant increase. It is proposed to use an increase of $0.35 \mu \mathrm{g} / \mathrm{L}$ per year to distinguish between patients with and those without prostate cancer [20]. However, the expectation is that the distinction between patients with and patients without prostate cancer will not be very satisfactory unless the application of the rule is limited to very low initial PSA values.

\section{References}

1. Börgermann $\mathrm{C}$, Rübben $\mathrm{H}$. Früherkennung des Prostatakarzinoms. Dtsch Ärzteblatt 2006;103:A2399-405.

2. Stenman UH, Paus E, Allard WJ, Anderson I, Andres C, et al. Summary report of the TD3 workshop: characterization of 83 antibodies against prostate-specific antigen. Tumour Biol 1999;20:1-12.

3. Linton HJ, Marks LS, Millar LS, Knott CL, Rittenhouse HG, Mikolajczyk SD. Benign prostate-specific antigen (BPSA) is increased in benign prostate disease. Clin Chem 2003;49: 253-9.

4. Stephan C, Meyer H, Paul EM, Kristiansen G, Loening SA, Lein $M$, et al. Serum $(-5,-7)$ proPSA for distinguishing stage and grade of prostate cancer. Anticancer Res 2007;27:18336.

5. Baumgart Y, Otto A, Schäfer A, Usbeck E, Cott C, Schott A, et al. Characterization of novel monoclonal antibodies for prostate-specific antigen (PSA) with potency to recognize PSA bound to alpha 2-macroglobulin. Clin Chem 2005;51: 84-92.

6. Reinauer H, Wood WG. External quality assessment for tumour marker analysis: state of the art and consequences for estimating diagnostic sensitivity and specificity. GMS Ger Med Sci 2005;3:Doc02 (20050530).

7. Passing $\mathrm{H}$, Bablok $\mathrm{W}$. A new biometrical procedure for testing the equality of measurements from two different analytical methods. Application of linear regression procedures for method comparison studies in clinical chemistry. J Clin Chem Clin Biochem 1983;21:709-20.

8. Arzideh F. Dissertation. Universität Bremen, 2008.

9. Haeckel R, Wosniok W, Arzideh F. A plea for interlaboratory reference limits. Part 1: General considerations and concepts for determination. Clin Chem Lab Med 2007:45;103342.

10. Arzideh F, Wosniok W, Gurr E, Hinsch W, Schumann G, Weinstock $\mathrm{N}$, et al. A plea for interlaboratory reference limits. Part 2: A bimodal retrospective concept for determining reference limits for from intralaboratory databases demonstrated by catalytic activity concentrations of enzymes. Clin Chem Lab Med 2007:45;1043-57.

11. Catalona WJ, Smith DS, Ratliff TL, Dodds KM, Coplen DE, Yuan JJ, et al. Measurement of prostate-specific antigen in serum as a screening test for prostate cancer. $\mathrm{N}$ Engl $\mathrm{J}$ Med 1991;324:1156-61.

12. Schroeder FA, Wildhagen MF. Low levels of PSA predict long-term risk of prostate cancer: results of the Baltimore longitudinal study of aging. Urology 2002;59:462-5.

13. Oesterling JE, Martin SK, Bergstrahl EJ, Lowe FC. The use of prostate-specific antigen in staging patients with newly diagnosed prostate cancer. J Am Med Assoc 1993;269: 57-60.

14. Schroeder FA, van der Cruijsen-Koeter I, de Koning HJ, Vis AN, Hoedemaeker RF, Kranse R. Prostate cancer detection at low prostate specific antigen. J Urol 2000;163:806-12.

15. Rohde V, Katalinic A, Wasem J. Gesundheitsberichterstattung des Bundes. Heft 36 Prostataerkrankungen. RKI Mitteilungen 2006.

16. Verhamme JM, Dieleman JP, Bleumink GS, van der Lei J, Sturkenboom MC, Artibani W, et al. Incidence and prevalence of lower urinary tract symptoms suggestive of benign prostatic hyperplasia in primary care - the triumph project. Euro Urol 2002;42:323-8.

17. Ricos C, et al. www.westgard.com/biodatabase1.htm

18. Krönig F. Dissertation (in preparation).

19. Reinauer H. Personal communications.

20. www.nccn.org/professionals/physician_gls/PDF/prostate detection.pdf, page 9. 